\title{
THE SUBRING OF GROUP COHOMOLOGY CONSTRUCTED BY PERMUTATION REPRESENTATIONS
}

\author{
DAVID J. GREEN ${ }^{1}$, IAN J. LEARY ${ }^{2}$ AND BJÖRN SCHUSTER ${ }^{1}$ \\ ${ }^{1}$ Department of Mathematics, University of Wuppertal, D-42097 Wuppertal, Germany \\ ${ }^{2}$ Faculty of Mathematical Studies, University of Southampton, \\ Southampton SO17 1BJ, UK
}

(Received 28 September 2000)

\begin{abstract}
Each permutation representation of a finite group $G$ can be used to pull cohomology classes back from a symmetric group to $G$. We study the ring generated by all classes that arise in this fashion, describing its variety in terms of the subgroup structure of $G$.

We also investigate the effect of restricting to special types of permutation representations, such as $\mathrm{GL}_{n}\left(\mathbb{F}_{p}\right)$ acting on flags of subspaces.
\end{abstract}

Keywords: group cohomology; finite groups; subgroup complexes; permutation representations

AMS 2000 Mathematics subject classification: Primary 20J06

\section{Introduction}

Throughout this paper $G$ shall denote a finite group, $p$ shall denote a prime number, and we shall write $\mathrm{H}^{*}(G)=\mathrm{H}^{*}\left(G, \mathbb{F}_{p}\right)$ for the cohomology ring of $G$ with coefficients in the field of $p$ elements. Each action of $G$ on a finite set $X$ gives rise to a homomorphism from $G$ to the symmetric group on $X, \Sigma(X)$, and hence a ring homomorphism from $\mathrm{H}^{*}(\Sigma(X))$ to $\mathrm{H}^{*}(G)$. Elements of $\mathrm{H}^{*}(G)$ in the image of this homomorphism could be called characteristic classes for the $G$-set $X$. For example, the characteristic classes defined by Segal and Stretch in [6] arise in this way. Our aim is to study the subring of $\mathrm{H}^{*}(G)$ generated by all such characteristic classes, for all finite $G$-sets $X$, which we shall denote by $S_{h}=S_{h}(G)$. In fact, our methods apply more generally. For a family $\mathcal{F}$ of subgroups of $G$, let $S_{\mathcal{F}}=S_{\mathcal{F}}(G)$ stand for the subring of $\mathrm{H}^{*}(G)$ generated by characteristic classes for $G$-sets for which the point stabilizers lie in $\mathcal{F}$. Under mild conditions on $\mathcal{F}$ we describe the variety for $S_{\mathcal{F}}$, by which we mean the functor from algebraically closed fields of characteristic $p$ to topological spaces that sends $k$ to the set of homomorphisms from $S_{\mathcal{F}}$ to $k$. We rely upon work in [4], which in turn relies on work of Quillen [5].

In [5], Quillen described the variety for $\mathrm{H}^{*}(G)$. Note that the variety is a covariant functor of $G$. It is easily described in the case when $G$ is elementary abelian (i.e. is abelian of exponent $p$ ). For general $G$, Quillen showed that the variety may be built up from the varieties for the elementary abelian subgroups of $G$. More formally, Quillen 
identified the variety as a colimit (of the variety functor) over a category with objects the elementary abelian subgroups of $G$ and morphisms those group homomorphisms induced by conjugation in $G$.

In [4], two of the current authors gave a generalization of Quillen's theorem to subrings of $\mathrm{H}^{*}(G)$ that are both 'large' and 'natural'. For such rings, they obtained a description of the variety as the colimit over a category with the same objects as Quillen's category, but (in general) more morphisms. The main example considered in [4] is the Chern subring, which is the subring of $\mathrm{H}^{*}(G)$ generated by the Chern classes of all unitary representations of $G$. Other examples include the subring generated by Chern classes of those representations realizable over a given subfield of the complex numbers.

It transpires that the rings $S_{\mathcal{F}}(G)$ are 'natural' and are 'large' provided that no element of $G$ of order $p$ is contained in every member of $\mathcal{F}$. In Theorem 2.6 we apply the results of [4] to give a description of the variety for $S_{\mathcal{F}}$ in terms of the group structure of $G$. In Corollary 2.9 we characterize those groups $G$ and families $\mathcal{F}$ for which the inclusion of $S_{\mathcal{F}}$ in $\mathrm{H}^{*}(G)$ is an inseparable isogeny. (Recall that an inseparable isogeny is a homomorphism inducing an isomorphism of varieties.) The map from $S_{h}$ to $\mathrm{H}^{*}(G)$ is not in general an inseparable isogeny. However, in Corollary 3.4 we show that this map always induces a bijection between the irreducible components of the two varieties. In terms of ideals, this is equivalent to the statement that for any $G$, distinct minimal prime ideals of $\mathrm{H}^{*}(G)$ have distinct intersections with $S_{h}$. By way of a contrast, there are examples (see [4] or Example 3.13 below) of groups $G$ for which the Chern ring does not separate the minimal primes of $\mathrm{H}^{*}(G)$.

Most of the work in this paper consists of an extended example. In $\S 4$ we specialize to the case when $G$ is the general linear group $\mathrm{GL}_{n}\left(\mathbb{F}_{p}\right)$, and compare the varieties for $\mathrm{H}^{*}(G), S_{h}$ and $S_{\pi}$, where $\pi$ denotes the family of parabolic subgroups of $G$. Equivalently, $S_{\pi}$ is the subring of $\mathrm{H}^{*}(G)$ generated by the characteristic classes for the permutation actions of $G=\mathrm{GL}_{n}\left(\mathbb{F}_{p}\right)$ on the various types of partial flags in $\mathbb{F}_{p}^{n}$. In particular, we show that for large even values of $n$, neither the inclusion of $S_{\pi}$ in $S_{h}$ nor the inclusion of $S_{h}$ in $\mathrm{H}^{*}(G)$ is an inseparable isogeny. Using the results of the previous two sections, this amounts to comparing three categories whose objects are the elementary abelian subgroups of $G$, and showing that, for large even $n$, these categories do not contain the same morphisms. The work in this section was motivated by a question posed by Fred Cohen, which formed the starting point for our work.

\section{Definitions and our main theorem}

First we describe the object of study precisely.

Definition 2.1. A non-empty family $\mathcal{F}$ of subgroups of $G$ will be called admissible if it is closed under conjugation in $G$, and the subgroup $\bigcap_{H \in \mathcal{F}} H$ of $G$ is a $p^{\prime}$-group. A $G$-set $X$ will be called an $\mathcal{F}$-set if each point stabilizer belongs to $\mathcal{F}$.

In particular, the family $\mathcal{F}_{h}$ consisting of all subgroups of $G$ is admissible, and all $G$-sets are $\mathcal{F}_{h}$-sets. 
Definition 2.2. Given a $G$-set $X$ of cardinality $n$, a choice of bijection between $X$ and the set $\{1, \ldots, n\}$ induces a homomorphism $\rho_{X}: G \rightarrow \Sigma_{n}$. For fixed $X$, any two choices of $\rho_{X}$ differ by an inner automorphism of $\Sigma_{n}$, and so the ring homomorphism $\rho_{X}^{*}: \mathrm{H}^{*}\left(\Sigma_{n}\right) \rightarrow \mathrm{H}^{*}(G)$ depends only on $X$ and not on the choice of bijection. Define $S_{\mathcal{F}}$ as the subring of $\mathrm{H}^{*}(G)$ generated by all $\operatorname{Im}\left(\rho_{X}^{*}\right)$ with $X$ an $\mathcal{F}$-set.

We shall now determine the variety of this ring $S_{\mathcal{F}}$. The following definition is needed to state the result.

Definition 2.3. Denote by $\mathcal{A}_{\mathcal{F}}$ the category whose objects are the elementary abelian $p$-subgroups of $G$, with $\mathcal{A}_{\mathcal{F}}(V, W)$ the set of injective group homomorphisms $f: V \rightarrow W$ satisfying: for every $H \in \mathcal{F}$ the $V$-sets $f^{!}(G / H)$ and $G / H$ are isomorphic. Here $f^{!}(G / H)$ means the following action of $V$ on $G / H$ :

$$
k * g H=f(k) g H .
$$

Remark 2.4. Compare this with the definition of the Quillen category, $\mathcal{A}(G)$, in [5]. This has the same objects as $\mathcal{A}_{\mathcal{F}}(G)$, and morphisms those group homomorphisms $f: V \rightarrow W$ such that for some $g \in G, f$ is equal to conjugation by $g$. It follows from the definitions that $\mathcal{A}(G)$ is a subcategory of $\mathcal{A}_{\mathcal{F}}$ for any family $\mathcal{F}$.

Remark 2.5. The category $\mathcal{A}_{\mathcal{F}_{h}}$ is identified in Lemma 3.2 .

Recall that the variety $\operatorname{var}(R)$ of a connected graded commutative $\mathbb{F}_{p}$-algebra $R$ is the functor that assigns to each algebraically closed field $k$ the topological space of ring homomorphisms from $R$ to $k$ with the Zariski topology.

Theorem 2.6. The cohomology ring $\mathrm{H}^{*}(G)$ is finitely generated as a module over $S_{\mathcal{F}}$, for any admissible family $\mathcal{F}$. Moreover, the restriction maps in cohomology induce a natural homeomorphism

$$
\underset{V \in \mathcal{A}_{\mathcal{F}}}{\operatorname{colim}} \operatorname{var}\left(\mathrm{H}^{*}(V)\right) \cong \operatorname{var}\left(S_{\mathcal{F}}\right) .
$$

Proof. Let $H_{1}, \ldots, H_{r}$ be a full set of class representatives for the conjugation action of $G$ on $\mathcal{F}$. Let $X$ be the $G$-set $\left(G / H_{1}\right) \amalg \cdots \amalg\left(G / H_{r}\right)$, and $n=|X|$. Then $X$ is an $\mathcal{F}$-set, and the kernel of the associated group homomorphism $\rho: G \rightarrow \Sigma_{n}$ is a $p^{\prime}$-group by admissibility.

Now compose $\rho$ with the regular representation $\operatorname{reg}_{\Sigma_{n}}$ of $\Sigma_{n}$ in the unitary group $U(n !)$. We obtain a degree $n$ ! representation of $G$, whose restriction to a Sylow $p$-subgroup $P$ of $G$ is a direct sum of copies of the regular representation. In particular, it is a faithful representation of $P$. The Chern classes of $\operatorname{reg}_{\Sigma_{n}} \circ \rho$ lie in $S_{\mathcal{F}}$ as they are images under $\rho^{*}$. Hence, by Venkov's proof [7] of the Evens-Venkov theorem, $H^{*}(P)$ is finitely generated as a module over $S_{\mathcal{F}}$. Therefore $\mathrm{H}^{*}(G)$ is finitely generated too.

This representation $\operatorname{reg}_{\Sigma_{n}} \circ \rho$ also restricts to every elementary abelian $p$-subgroup of $G$ as a (non-zero) direct sum of copies of the regular representation, and so is $p$-regular in the sense of [4]. So $S_{\mathcal{F}}$ contains the Chern classes of a $p$-regular representation. Moreover, the $\operatorname{ring} S_{\mathcal{F}}$ is clearly homogeneously generated and closed under the action of the Steenrod algebra. By Theorem 6.1 of [4] it follows firstly that $\operatorname{var}\left(S_{\mathcal{F}}\right)$ is a colimit of the desired 
form over some category of elementary abelians, and secondly that Lemma 2.7 identifies this category as being $\mathcal{A}_{\mathcal{F}}$.

Lemma 2.7. Let $f: V \rightarrow W$ be an injective group homomorphism between elementary abelian subgroups of $G$. Then $f$ lies in $\mathcal{A}_{\mathcal{F}}$ if and only if for every $x \in S_{\mathcal{F}}$, the class $\operatorname{Res}_{V}^{G}(x)-f^{*} \operatorname{Res}_{W}^{G}(x)$ lies in the nilradical of $\mathrm{H}^{*}(V)$.

Proof. Suppose $f \in \mathcal{A}_{\mathcal{F}}$. Pick any $\mathcal{F}$-set $Y$, and let $\rho: G \rightarrow \Sigma_{|Y|}$ be the associated group homomorphism. Since the $V$-sets $Y$ and $f^{!}(Y)$ are isomorphic, $f$ induces a map $\rho(V) \rightarrow \rho(W)$, and this map is equal to conjugation by some $\sigma \in \Sigma_{|Y|}$. Hence $\operatorname{Res}_{V}^{G}-f^{*} \operatorname{Res}_{W}^{G}$ kills $\operatorname{Im}\left(\rho^{*}\right)$.

Conversely, suppose that $f \notin \mathcal{A}_{\mathcal{F}}$. Recall that in the proof of Theorem 2.6 we constructed an $\mathcal{F}$-set $X$, such that the kernel of the associated group homomorphism $\rho: G \rightarrow$ $\Sigma_{|X|}$ is a $p^{\prime}$-group. By assumption on $f$, there is some $H \in \mathcal{F}$ such that the $V$-sets $f^{!}(G / H), G / H$ are not isomorphic. Define $Y$ by

$$
Y= \begin{cases}X \amalg(G / H) & \text { if } f^{!}(X), X \text { are isomorphic as } V \text {-sets, } \\ X & \text { otherwise. }\end{cases}
$$

Then $Y$ is an $\mathcal{F}$-set and $V$ acts faithfully on $Y, f^{!}(Y)$, but these two $V$-sets are not isomorphic.

We have thus constructed embeddings of $V$ and $W$ in $\Sigma_{|Y|}$, such that $f$ is not induced by conjugation in $\Sigma_{|Y|}$. Therefore there is a class $\xi \in \mathrm{H}^{*}\left(\Sigma_{|Y|}\right)$ such that

$$
\operatorname{Res}_{V}^{\Sigma_{|Y|}}(\xi)-f^{*} \operatorname{Res}_{W}^{\Sigma_{|Y|}}(\xi)
$$

is not nilpotent (apply the results of $[4, \S 9]$ to the group $\Sigma_{|Y|}$ ). Moreover, these embeddings of $V, W$ in $\Sigma_{|Y|}$ factor through $G \rightarrow \Sigma_{|Y|}$. Pulling $\xi$ back to $\mathrm{H}^{*}(G)$, we get the desired class.

Remark 2.8. Theorem 2.6 may be compared with Quillen's theorem (see $[\mathbf{2}, \S 9.2]$ or [5]), which states that the restriction maps induce a natural isomorphism

$$
\underset{V \in \mathcal{A}}{\operatorname{colim}} \operatorname{var}\left(\mathrm{H}^{*}(V)\right) \cong \operatorname{var}\left(\mathrm{H}^{*}(G)\right) .
$$

Corollary 2.9. The inclusion of $S_{\mathcal{F}}$ in $\mathrm{H}^{*}(G)$ is an inseparable isogeny if and only if the category $\mathcal{A}_{\mathcal{F}}$ is equal to the Quillen category $\mathcal{A}$. If the family $\mathcal{F}_{1}$ is contained in $\mathcal{F}_{2}$, the inclusion of $S_{\mathcal{F}_{1}}$ in $S_{\mathcal{F}_{2}}$ is an inseparable isogeny if and only if $\mathcal{A}_{\mathcal{F}_{1}}=\mathcal{A}_{\mathcal{F}_{2}}$.

Proof. This is just a special case of Corollary 6.4 of [4].

\section{Examples}

Definition 3.1. We define the hereditary category $\mathcal{A}_{h}$ of $G$ to be $\mathcal{A}_{\mathcal{F}_{h}}$, where $\mathcal{F}_{h}$ is the admissible family of all subgroups of $G$. Write $S_{h}$ for $S_{\mathcal{F}_{h}}$.

Recall that $\sim_{G}$ denotes the equivalence relation conjugacy in $G$. 
Lemma 3.2. Let $f: V \rightarrow W$ be an injective group homomorphism between elementary abelian subgroups of $G$. Then $f$ lies in $\mathcal{A}_{h}$ if and only if $f(U) \sim_{G} U$ for every elementary abelian $U \leqslant V$.

Let $\mathcal{F}$ be an admissible family containing all non-trivial elementary abelian $p$-subgroups of $G$. Then $\mathcal{A}_{\mathcal{F}}=\mathcal{A}_{h}$.

Remark 3.3. This property of $\mathcal{A}_{h}$ is the reason for the name hereditary.

Proof. We prove that the first part holds for any $\mathcal{F}$ satisfying the conditions of the second part, not just for $\mathcal{F}_{h}$.

First suppose that $U$ is a subgroup of $V$ and $f(U) \chi_{G} U$. Then the $V$-set $G / U$ has a point stabilized by $U$, but $f^{!}(G / U)$ does not. Hence these two $V$-sets are not isomorphic, and so $f$ does not lie in $\mathcal{A}_{\mathcal{F}}$.

For the if part, consider any $H \in \mathcal{F}$ and any $U \leqslant V$. The coset $g H$ is fixed by $U$ if and only if $U^{g} \leqslant H$. Since $f(U) \sim_{G} U$, the number of $U$-fixed points in $f^{!}(G / H)$ is the same as for $G / H$. It follows that the $V$-sets $f^{!}(G / H)$ and $G / H$ are isomorphic.

In $[4, \S 9]$, a category $\mathcal{C}$ consisting of elementary abelian subgroups of $G$ and injective group homomorphisms was defined to be closed if the following three conditions are satisfied: the Quillen category $\mathcal{A}$ is a subcategory; isomorphisms lie in $\mathcal{C}$ if and only if their inverses do; and $f_{\mid U}: U \rightarrow f(U)$ lies in $\mathcal{C}$ for every $f: V \rightarrow W$ in $\mathcal{C}$ and every $U \leqslant V$.

Corollary 3.4. Objects of $\mathcal{A}_{h}$ are isomorphic if and only if they are conjugate as subgroups of $G$. In fact, $\mathcal{A}_{h}$ is the unique largest category of elementary abelian subgroups of $G$ which is closed in the sense of $[\mathbf{4}, \S 9]$, and in which objects are isomorphic if and only if they are conjugate as subgroups of $G$.

Proof. It follows from the definition of $\mathcal{A}_{\mathcal{F}}$ that $\mathcal{A}_{\mathcal{F}}$ is closed for every admissible family $\mathcal{F}$. The result follows from Lemma 3.2.

Remark 3.5. For an elementary abelian $p$-group $V \leqslant G$, the classes in $\mathrm{H}^{*}(G)$ with nilpotent restriction to $V$ constitute a prime ideal $\mathfrak{p}_{V}$. It follows from Quillen's theorem that the $\mathfrak{p}_{M}$ with $M \leqslant G$ maximal elementary abelian are the minimal prime ideals in $\mathrm{H}^{*}(G)$. Similarly, Theorem 2.6 means that the $\mathfrak{p}_{M} \cap S_{h}$ are the minimal prime ideals in $S_{h}$.

So, by the first part of Corollary 3.4, 'intersection with $S_{h}$ ' induces a bijection from the minimal primes of $\mathrm{H}^{*}(G)$ to those of $S_{h}$. Put geometrically, the irreducible components of $\operatorname{var}\left(\mathrm{H}^{*}(G)\right)$ and of $\operatorname{var}\left(S_{h}\right)$ are in natural one-to-one correspondence.

Definition 3.6. Let $G$ be the general linear group $\mathrm{GL}_{n}\left(\mathbb{F}_{p}\right)$. We define the parabolic category $\mathcal{A}_{\pi}$ to be $\mathcal{A}_{\mathcal{F}_{\pi}}$, where $\mathcal{F}_{\pi}$ is the collection of all parabolic subgroups of $G$. Write $S_{\pi}$ for $S_{\mathcal{F}_{\pi}}$.

Proposition 3.7. The parabolic category is admissible. We have

$$
\operatorname{var}\left(S_{h}\right) \cong \underset{V \in \mathcal{A}_{h}}{\operatorname{colim}} \operatorname{var}\left(\mathrm{H}^{*}(V)\right) \quad \text { and } \quad \operatorname{var}\left(S_{\pi}\right) \cong \underset{V \in \mathcal{A}_{\pi}}{\operatorname{colim}} \operatorname{var}\left(\mathrm{H}^{*}(V)\right) .
$$


Proof. The upper triangular matrices constitute a parabolic subgroup, as do the lower triangular matrices. These two groups intersect in a $p^{\prime}$-group, so $\mathcal{F}_{\pi}$ is admissible. Apply Theorem 2.6 for the admissible families $\mathcal{F}_{h}$ and $\mathcal{F}_{\pi}$.

Example 3.8. Let $p$ be an odd prime, and let $1<q<p$. For any finite group $G$ and any elementary abelian $V \leqslant G$, the automorphism $v \mapsto v^{q}$ of $V$ lies in $\mathcal{A}_{h}$ by Lemma 3.2. But in general this map does not lie in $\mathcal{A}$. An example is when $G$ is abelian (and not a $p^{\prime}$-group). For such groups, the inclusion of $S_{h}$ in $\mathrm{H}^{*}(G)$ in not an inseparable isogeny.

Example 3.9. In Corollary 4.4, we shall see that for $n \geqslant 3$ and $G$ the group $\mathrm{GL}_{2 n}\left(\mathbb{F}_{p}\right)$, there is a rank two elementary abelian subgroup $E$ of $G$ such that not all automorphisms of $E$ lie in $\mathcal{A}$; and yet all non-trivial elements of $E$ are conjugate in $G$, which means that all automorphisms of $E$ lie in $\mathcal{A}_{h}$. Hence the inclusion of $S_{h}$ in $\mathrm{H}^{*}(G)$ is not an inseparable isogeny.

Example 3.10. In Theorem 4.6, we shall see that for $n \geqslant 6$ and $G=\mathrm{GL}_{2 n}\left(\mathbb{F}_{p}\right)$, there are non-conjugate rank two elementary abelian subgroups of $G$ which are isomorphic in $\mathcal{A}_{\pi}$. Hence the varieties of $S_{\pi}, S_{h}$ and $\mathrm{H}^{*}(G)$ are all distinct.

Example 3.11. The elementary abelian $p$-subgroups of $G$ form an admissible family, as do all $p$-subgroups of $G$. If $G$ has $p$-rank at least two, then we can omit the trivial subgroup in both families.

In all these cases, the category $\mathcal{A}_{\mathcal{F}}$ is equal to $\mathcal{A}_{h}$ by Lemma 3.2 . Hence inclusion of $S_{\mathcal{F}}$ in $S_{h}$ is an inseparable isogeny.

Example 3.12. Alperin [1] defines a subgroup $H$ of an abstract finite group $G$ to be $p$-parabolic if $H=N_{G}\left(O_{p}(H)\right)$. (Recall that $O_{p}(H)$ is defined to be the largest normal $p$ subgroup of $H$.) For $G=\mathrm{GL}_{n}\left(\mathbb{F}_{p}\right)$, this coincides with the usual definition of a parabolic subgroup as the stabilizer of a flag. He also defines a $p$-subgroup $H$ of $G$ to be $p$-radical if $H=O_{p}\left(N_{G}(H)\right)$. Hence the $p$-parabolic subgroups are the normalizers of the $p$-radical subgroups. Note that algebraic topologists sometimes use the term ' $p$-stubborn' instead of ' $p$-radical'.

If $O_{p}(G)=1$, then the parabolic subgroups and the $p$-radical subgroups each form admissible families, since Sylow $p$-subgroups are $p$-radical and $O_{p}(G)$ is the intersection of all Sylow $p$-subgroups.

For $p=11$, the sporadic finite simple group $J_{4}$ has the trivial intersection property: distinct Sylow $p$-subgroups intersect trivially. Hence the parabolic subgroups are the admissible family consisting of $J_{4}$ itself and the Sylow normalizers. The action of any order $p$ cyclic subgroup on cosets of a Sylow normalizer has one fixed point, with the remaining orbits having length $p$. As there are two distinct conjugacy classes of order $p$ cyclics, the parabolic category is larger than the hereditary category. The cohomology of $J_{4}$ at the prime 11 was computed in [3].

Example 3.13. In general the subring $S_{h}$ is far larger than the subring generated by Chern classes of permutation representations: i.e. the subring generated by all images of $\mathrm{H}^{*}(\mathrm{BU}(n))$ under homomorphisms $G \rightarrow \Sigma_{n} \rightarrow U(n)$, where $\Sigma_{n}$ is embedded in $U(n)$ as 
the permutation matrices. In general, neither $S_{h}$ nor the whole Chern subring is contained in the other.

In [4] it was shown that the varieties for the Chern subring and for the subring generated by Chern classes of permutation representations are colimits over the categories $\mathcal{A}^{\prime}$ and $\mathcal{A}_{P}$, respectively, where $f: V \rightarrow W$ lies in $\mathcal{A}^{\prime}$ if and only if $f(v) \sim_{G} v$ for every element $v \in V$, and lies in $\mathcal{A}_{P}$ if and only if $f(U) \sim_{G} U$ for every cyclic subgroup of $V$.

When $p=2, \mathcal{A}^{\prime}$ and $\mathcal{A}_{P}$ are equal for any $G$. When $p$ is odd, and $G$ is cyclic of order $p$, $\mathcal{A}_{P}=\mathcal{A}_{h}$, and both are properly contained in $\mathcal{A}^{\prime}$. For any prime $p$, there are elementary abelian $p$-groups of rank two in the general linear group $\mathrm{GL}_{3}\left(\mathbb{F}_{p}\right)$ that are not conjugate (and hence not isomorphic in $\mathcal{A}_{h}$ ), but are isomorphic in $\mathcal{A}^{\prime}$ and in $\mathcal{A}_{P}$. See $[4, \S 7]$ for a discussion of this example.

\section{An extended example}

Fred Cohen asked the third author about the subring of $\mathrm{H}^{*}\left(\mathrm{GL}_{n}\left(\mathbb{F}_{p}\right)\right)$ generated by the permutation representations on flags. In our language, the question concerns the subring $S_{\pi}$. This question provided the starting point for the current paper. We provide a partial answer to this question by comparing the varieties for $\mathrm{H}^{*}\left(\mathrm{GL}_{n}\left(\mathbb{F}_{p}\right)\right), S_{h}$ and $S_{\pi}$, which is equivalent to comparing the categories $\mathcal{A}, \mathcal{A}_{h}$ and $\mathcal{A}_{\pi}$. Recall that there are inclusions

$$
\mathcal{A} \subseteq \mathcal{A}_{h} \subseteq \mathcal{A}_{\pi} .
$$

Let $G$ be the general linear group $\mathrm{GL}_{2 n}\left(\mathbb{F}_{p}\right)$. We show that all three categories are distinct for $n \geqslant 6$. The most time-consuming part is showing that $\mathcal{A}_{\pi}$ differs from $\mathcal{A}_{h}$ for such $n$. By Corollary 3.4 it suffices to show that there are elementary abelian $p$-subgroups of $G$ which are isomorphic in $\mathcal{A}_{\pi}$ but not conjugate in $G$. We shall find rank two examples using modular representation theory.

Let $p$ be a prime number, and let $A, B$ be generators for the rank two elementary abelian $p$-group $V \cong C_{p} \times C_{p}$. To each matrix $J \in \mathrm{GL}_{n}\left(\mathbb{F}_{p}\right)$, there is an associated representation $\rho_{J}: V \rightarrow \mathrm{GL}_{2 n}\left(\mathbb{F}_{p}\right)$ defined by

$$
A \stackrel{\rho_{J}}{\mapsto}\left(\begin{array}{cc}
I & I \\
0 & I
\end{array}\right), \quad B \stackrel{\rho_{J}}{\mapsto}\left(\begin{array}{ll}
I & J \\
0 & I
\end{array}\right),
$$

where $I \in \mathrm{GL}_{n}\left(\mathbb{F}_{p}\right)$ is the identity matrix. The following lemma is well known in the modular representation theory of $V$.

Lemma 4.1. Let $J, J^{\prime} \in \mathrm{GL}_{n}\left(\mathbb{F}_{p}\right)$. Then the representations $\rho_{J}, \rho_{J^{\prime}}$ are isomorphic if and only if $J, J^{\prime}$ are conjugate in $\mathrm{GL}_{n}\left(\mathbb{F}_{p}\right)$.

Proof. The centralizer of

$$
\left(\begin{array}{ll}
I & I \\
0 & I
\end{array}\right)
$$


consists of all matrices of the form

$$
\left(\begin{array}{cc}
A & B \\
0 & A
\end{array}\right)
$$

The conjugate of

$$
\left(\begin{array}{ll}
I & J \\
0 & I
\end{array}\right)
$$

under such a matrix is

$$
\left(\begin{array}{ll}
I & J^{\prime} \\
0 & I
\end{array}\right)
$$

with $J^{\prime}=A J A^{-1}$.

Lemma 4.2. For any matrix $M \in \mathrm{GL}_{n}\left(\mathbb{F}_{p}\right)$, the matrices

$$
\left(\begin{array}{cc}
I & M \\
0 & I
\end{array}\right) \text { and }\left(\begin{array}{ll}
I & I \\
0 & I
\end{array}\right)
$$

are conjugate in $\mathrm{GL}_{2 n}\left(\mathbb{F}_{p}\right)$.

Proof. Conjugate on the right by

$$
\left(\begin{array}{cc}
M & 0 \\
0 & I
\end{array}\right)
$$

First we compare the categories $\mathcal{A}_{h}$ and $\mathcal{A}$.

Lemma 4.3. Suppose there is a primitive element $\theta \in \mathbb{F}_{p^{n}} / \mathbb{F}_{p}$ with minimal polynomial $f$ such that $\theta+1$ is not a root of $f$. Then the Quillen category $\mathcal{A}$ for $G=G_{2 n}\left(\mathbb{F}_{p}\right)$ is strictly smaller than the hereditary category $\mathcal{A}_{h}$.

Proof. Let $J \in \mathrm{GL}_{n}\left(\mathbb{F}_{p}\right)$ be the matrix in rational canonical form with characteristic polynomial $f$. (By this we mean the matrix with 1s below its diagonal, minus the coefficients of $f$ along its final column and zeros elsewhere, but in fact any matrix with characteristic polynomial $f$ will suffice.) Since $f$ is irreducible, $J$ has no eigenvalues in $\mathbb{F}_{p}$. In particular, this means that $I+J$ lies in $\mathrm{GL}_{n}\left(\mathbb{F}_{p}\right)$. The condition on the roots of $f$ means that $J$ and $I+J$ have distinct characteristic polynomials, and so are non-conjugate in $\mathrm{GL}_{n}\left(\mathbb{F}_{p}\right)$.

Let $E$ be $\operatorname{Im}\left(\rho_{J}\right)$, the rank two elementary abelian generated by $a=\rho_{J}(A)$ and $b=$ $\rho_{J}(B)$. Hence

$$
a=\left(\begin{array}{cc}
I & I \\
0 & I
\end{array}\right), \quad b=\left(\begin{array}{cc}
I & J \\
0 & I
\end{array}\right), \quad a b=\left(\begin{array}{cc}
I & I+J \\
0 & I
\end{array}\right) .
$$


Let $\phi$ be the automorphism of $E$ which fixes $a$ and sends $b$ to $a b$. By the proof of Lemma 4.1 we see that $\phi \notin \mathcal{A}$, since $J$ and $I+J$ are not conjugate. To see that $\phi \in \mathcal{A}_{h}$, it suffices by Lemma 3.2 to show that $e, \phi(e)$ are conjugate in $G=\mathrm{GL}_{2 n}\left(\mathbb{F}_{p}\right)$ for each non-trivial $e \in E$. But this follows from Lemma 4.2.

Corollary 4.4. Set $n_{0}=2$ for $p \geqslant 3$ and $n_{0}=3$ for $p=2$. For $G=\mathrm{GL}_{2 n}\left(\mathbb{F}_{p}\right)$ and $n \geqslant n_{0}$, the Quillen category $\mathcal{A}$ is strictly smaller than the hereditary category $\mathcal{A}_{h}$.

Proof. We show that there is a $\theta$ satisfying the conditions of Lemma 4.3. The Galois group of $\mathbb{F}_{p^{n}} / \mathbb{F}_{p}$ is cyclic of order $n$, generated by the Frobenius automorphism. Hence $\theta \in \mathbb{F}_{p^{n}}$ has the same minimal polynomial as $\theta+1$ if and only if $\theta$ is a root of $x^{p^{m}}-x-1$ for some $m<n$. Therefore there are at least $p^{n}-p^{n-1}-p^{n-2}-\cdots-p$ elements $\theta$ of $\mathbb{F}_{p^{n}}$ such that $\theta, \theta+1$ do not have the same minimal polynomial. If $p \geqslant 3$ and $n \geqslant 2$, then this exceeds $p^{n-1}$, and there are at most $p^{n-1}$ non-primitive elements of $\mathbb{F}_{p^{n}} / \mathbb{F}_{p}$ : hence there exists a $\theta$ of the required form.

Now suppose that $p$ is 2 . The roots of $x^{2^{m}}-x-1$ all lie in $\mathbb{F}_{2^{2 m}}$, and so can only be primitive elements of $\mathbb{F}_{2^{n}} / \mathbb{F}_{2}$ if $n \mid 2 m$. Since $m<n$, this can only happen if $n=2 m$. So the number of $\theta \in \mathbb{F}_{2^{n}} / \mathbb{F}_{2}$ such that $\theta, \theta+1$ have distinct minimal polynomials exceeds $2^{n-1}$ provided $n>2$, and there are at most $2^{n-1}$ non-primitives. Again, the required $\theta$ exists.

Now we compare the categories $\mathcal{A}_{\pi}$ and $\mathcal{A}_{h}$. To each irreducible degree $n$ monic polynomial $f \in \mathbb{F}_{p}[x]$ there is an associated $(n \times n)$-matrix $J_{f}$ in rational canonical form. Define the representation $\rho_{f}: V \rightarrow \mathrm{GL}_{2 n}\left(\mathbb{F}_{p}\right)$ to be $\rho_{J_{f}}$. By Lemma 4.1, distinct $f$ give rise to non-isomorphic representations.

Proposition 4.5. Let $H$ be a parabolic subgroup of $\mathrm{GL}_{2 n}\left(\mathbb{F}_{p}\right)$, and let $f$ be an irreducible degree $n$ polynomial. The embedding $\rho_{f}$ turns $G / H$ into a $V$-set. The isomorphism type of this $V$-set does not depend on $f$.

Theorem 4.6. Set $n_{0}=5$ for $p \geqslant 5$ and $n_{0}=6$ for $p=2,3$. For $G=\mathrm{GL}_{2 n}\left(\mathbb{F}_{p}\right)$ and $n \geqslant n_{0}$, there are rank two elementary abelian subgroups of $G$ which are isomorphic in the parabolic category $\mathcal{A}_{\pi}$ but are not conjugate in $G$, and therefore are not isomorphic in $\mathcal{A}_{h}$.

Proof. Recall from Corollary 3.4 that elementary abelian subgroups are isomorphic in $\mathcal{A}_{h}$ if and only if they are conjugate in $G$.

For any pair $f, g$ of irreducible degree $n$ monic polynomials over $\mathbb{F}_{p}$, the isomorphism

$$
\rho_{g} \circ \rho_{f}^{-1}: \operatorname{Im}\left(\rho_{f}\right) \rightarrow \operatorname{Im}\left(\rho_{g}\right)
$$

lies in $\mathcal{A}_{\pi}$ by Proposition 4.5. As distinct irreducible polynomials give rise to nonisomorphic representations, the number of irreducible $g$ such that $\operatorname{Im}\left(\rho_{g}\right)$ is conjugate to a given $\operatorname{Im}\left(\rho_{f}\right)$ cannot exceed $|\operatorname{Aut}(V)|=\left(p^{2}-1\right)\left(p^{2}-p\right)$. But for $n \geqslant n_{0}$ there are always more irreducibles than this. For the total number of irreducibles is equal to $\pi_{n} / n$, where $\pi_{n}$ is the number of primitive elements in $\mathbb{F}_{p^{n}} / \mathbb{F}_{p}$. We have $\pi_{5}=p^{5}-p$, 
$\pi_{6}=p^{6}-p^{3}-p^{2}+p$ and $\pi_{n} \geqslant p^{n}-p^{n-2}$ for $n \geqslant 7$. It is then straightforward to check that $\pi_{n} / n>\left(p^{2}-1\right)\left(p^{2}-p\right)$ for $n \geqslant n_{0}$.

We now derive some results needed in the proof of Proposition 4.5. We take $f$ to be a degree $n$ irreducible polynomial over $\mathbb{F}_{p}$, and $J=J_{f}$ to be the associated matrix in rational canonical form.

Lemma 4.7. Let $W$ be a proper subspace of $\mathbb{F}_{p}^{n}$. Define $m, r$ by $m=\operatorname{dim}(W)$ and $m+r=\operatorname{dim}(W+J W)$. There is a partition $\lambda=\left(\lambda_{1}, \ldots, \lambda_{r}\right)$ of $m$ with length $r$ (so $\lambda_{r} \geqslant 1$ ) and elements $w_{1}, \ldots, w_{r}$ of $W$, such that

(1) the $J^{a} w_{i}$ for $1 \leqslant i \leqslant r$ and $0 \leqslant a \leqslant \lambda_{i}-1$ are a basis for $W$, and

(2) the $J^{a} w_{i}$ for $1 \leqslant i \leqslant r$ and $0 \leqslant a \leqslant \lambda_{i}$ are a basis for $W+J W$.

We call such an $r$-tuple $w_{1}, \ldots, w_{r}$ a $(J, \lambda)$-base for $W$.

Furthermore, $\lambda$ is uniquely determined by $J, W$; and the number of $(J, \lambda)$-bases for $W$ depends solely on $\lambda$.

Observe that $m+r \leqslant n$ and that $r \leqslant m$. Since $J$ is the rational canonical form associated to an irreducible polynomial, there are no $J$-invariant subspaces other than 0 and $\mathbb{F}_{p}^{n}$. Hence $r=0$ if and only if $m=0$.

Proof. The proof is by induction on $m$. The case $m=0$ is clear. Now suppose that $m>0$ and the result has been proved for $\operatorname{dim}(W) \leqslant m-1$. Set $W^{\prime}=W \cap J^{-1} W$, so $\operatorname{dim}\left(W^{\prime}\right)=m-r$. Define $r^{\prime}$ by $r^{\prime}=\operatorname{dim}\left(W^{\prime}+J W^{\prime}\right)-\operatorname{dim}\left(W^{\prime}\right)$.

As $m>0$ we have $m-r \leqslant m-1$, so we can apply the result to $W^{\prime}$. Thus we obtain a length $r^{\prime}$ partition $\lambda^{\prime}=\left(\lambda_{1}^{\prime}, \ldots, \lambda_{r^{\prime}}^{\prime}\right)$ of $m-r$ and an $r^{\prime}$-tuple $w_{1}^{\prime}, \ldots, w_{r^{\prime}}^{\prime} \in W^{\prime}$. For $1 \leqslant i \leqslant r^{\prime}$ set $\lambda_{i}=\lambda_{i}^{\prime}+1$ and $w_{i}=w_{i}^{\prime}$. Observe that

$$
\operatorname{dim}(W)-\operatorname{dim}\left(W^{\prime}+J W^{\prime}\right)=r-r^{\prime} .
$$

Pick a basis $w_{r^{\prime}+1}, \ldots, w_{r}$ for any complement of $W^{\prime}+J W^{\prime}$ in $W$, and set $\lambda_{i}=1$ for $r^{\prime}<i \leqslant r$. Then $\lambda$ is a length $r$ partition of $n$, and the $J^{a} w_{i}$ for $1 \leqslant i \leqslant r$ and $0 \leqslant a \leqslant \lambda_{i}-1$ are a basis for $W$.

Moreover, the $J^{\lambda_{i}^{\prime}} w_{i}^{\prime}$ for $1 \leqslant i \leqslant r^{\prime}$ are a basis for a complement of $W^{\prime}$ in $W^{\prime}+J W^{\prime}$; and $w_{r^{\prime}+1}, \ldots, w_{r}$ are a basis for a complement of $W^{\prime}+J W^{\prime}$ in $W$. Hence the $J^{\lambda_{i}-1} w_{i}$ for $1 \leqslant i \leqslant r$ are a basis for a complement of $W^{\prime}$ in $W$. By definition of $W^{\prime}$, this means that the $J^{\lambda_{i}} w_{i}$ for $1 \leqslant i \leqslant r$ are a basis for a complement of $W$ in $W+J W$. So the $w_{i}$ constitute a $(J, \lambda)$-base.

Conversely, suppose that $\mu$ is a partition of $m$ of length $r$, and that $v_{1}, \ldots, v_{r}$ is a $(J, \mu)$ base for $W$. The elements $J^{a} v_{i}$ for $0 \leqslant a \leqslant \mu_{i}-2$ are a basis for $W^{\prime}$, the $J^{\mu_{i}-1} v_{i}$ with $\mu_{i} \geqslant 2$ extend this to a basis for $W^{\prime}+J W^{\prime}$, and the $v_{i}$ with $\mu_{i}=1$ extend this to a basis for $W$. Hence the number of $i$ with $\mu_{i}=1$ is equal to $\operatorname{dim}(W)-\operatorname{dim}\left(W^{\prime}+J W^{\prime}\right)$. Passing to $W^{\prime}$, we deduce by induction that $\lambda$ and $\mu$ are equal; and that $\lambda$ alone determines the number of $(J, \lambda)$-bases $w_{1}, \ldots, w_{r}$. 
Lemma 4.8. Fix $J$ and fix partitions $\lambda, \lambda^{\prime}$. For any proper $W \subset \mathbb{F}_{p}^{n}$ with partition $\lambda$, the number of subspaces $W^{\prime}$ of $W$ with partition $\lambda^{\prime}$ depends solely on $\lambda, \lambda^{\prime}$.

Proof. Denote by $w_{i}, w_{i}^{\prime}$ the elements of a $(J, \lambda)$-base for $W, W^{\prime}$, respectively. Set $m=\operatorname{dim}(W)$ and $r=\operatorname{dim}(W+J W)-m$, as in Lemma 4.7.

Construct a basis $b_{1}, \ldots, b_{n}$ for $\mathbb{F}_{p}^{n}$ as follows:

(i) $b_{1}, \ldots, b_{m}$ is the basis $w_{1}, J w_{1}, \ldots, J^{\lambda_{1}-1} w_{1}, w_{2}, \ldots, J^{\lambda_{r}-1} w_{r}$ for $W$ given by Lemma 4.7;

(ii) $b_{m+1}, \ldots, b_{m+r}$ is the corresponding extension $J^{\lambda_{1}} w_{1}, \ldots, J^{\lambda_{r}} w_{r}$ to a basis for $W+$ $J W$; and

(iii) $b_{m+r+1}, \ldots, b_{n}$ is any extension to a basis for $\mathbb{F}_{p}^{n}$.

In the matrix of $J$ for this basis, the first $m$ columns describe the action on $W$, and depend solely on $\lambda$. So the number of $\left(J, \lambda^{\prime}\right)$-bases giving rise to a subspace of $W$ with partition $\lambda^{\prime}$ is independent of $J$. By Lemma 4.7, the number of $\left(J, \lambda^{\prime}\right)$-bases for any such $W^{\prime}$ depends solely on $\lambda^{\prime}$.

Corollary 4.9. Let $\lambda$ be a partition of $m<n$. The number of proper subspaces $W$ of $\mathbb{F}_{p}^{n}$ with partition $\lambda$ is independent of $f$.

Proof. The codimension 1 subspaces of $\mathbb{F}_{p}^{n}$ all have partition $(n-1)$ : so by Lemma 4.8 each contains the same number of such $W$, and this number is independent of $f$.

Corollary 4.10. Fix $0 \leqslant m_{0}<m_{1}<\cdots<m_{s}$ and partitions $\lambda^{i}$ of $m_{i}$. The number of flags $W_{0} \subset W_{1} \subset \cdots \subset W_{s}$ of proper subspaces of $\mathbb{F}_{p}^{n}$ in which $W_{i}$ has partition $\lambda^{i}$ is independent of $f$.

Proof. The case $s=1$ is Corollary 4.9. The general case is by induction on $s$ using Lemma 4.8

Proof of Proposition 4.5. We must show that for each parabolic subgroup $H \leqslant G$, the isomorphism class of the $V$-set structure induced on $G / H$ by $\rho_{f}$ does not depend on $f$. Now, two finite $V$-sets $X, Y$ are isomorphic if and only if for each subgroup $U$ of $V$, the sets $X^{U}, Y^{U}$ have the same cardinality.

The case $U=1$ is clear. For the cyclic subgroups, observe that since $J$ has no invariant subspaces and therefore no eigenvectors, the matrix $\lambda I+\mu J$ is invertible for all $(\lambda, \mu) \in$ $\mathbb{F}_{p}^{2} \backslash\{0\}$. Therefore, by Lemma 4.2, all non-trivial elements of $\operatorname{Im}\left(\rho_{f}\right)$ are conjugate in $\mathrm{GL}_{2 n}\left(\mathbb{F}_{p}\right)$ to each other, and so the number of fixed cosets is independent of $f$.

Only the hardest case remains to be proved: that the number of cosets fixed by $V$ itself is independent of $f$. Recall that the parabolic subgroups in $\mathrm{GL}_{2 n}$ are the flag stabilizers. Define the type of a flag

$$
X_{0} \subset X_{1} \subset \cdots \subset X_{t}
$$


of subspaces of $\mathbb{F}_{p}^{2 n}$ to be the $(t+1)$-tuple $\left(\operatorname{dim}\left(X_{0}\right), \ldots, \operatorname{dim}\left(X_{t}\right)\right)$. The flags of any given type are permuted transitively by $\mathrm{GL}_{2 n}\left(\mathbb{F}_{p}\right)$. Our task is to show that the number of $V$-invariant flags of any given type does not depend on the choice of irreducible polynomial $f$.

Associated to the block matrices is a splitting of $\mathbb{F}_{p}^{2 n}$ as $\mathbb{F}_{p}^{n} \oplus \mathbb{F}_{p}^{n}$. Let $i: \mathbb{F}_{p}^{n} \rightarrow \mathbb{F}_{p}^{2 n}$ be inclusion as the first factor, and $j: \mathbb{F}_{p}^{2 n} \rightarrow \mathbb{F}_{p}^{n}$ projection onto the second factor. Let $X$ be an invariant subspace of $\mathbb{F}_{p}^{2 n}$, and set $W=j(X), Z=i^{-1}(X)$. Then

$$
\left(\begin{array}{ll}
I & I \\
0 & I
\end{array}\right)\left(\begin{array}{c}
z \\
w
\end{array}\right)=\left(\begin{array}{c}
z+w \\
w
\end{array}\right), \quad\left(\begin{array}{ll}
I & J \\
0 & I
\end{array}\right)\left(\begin{array}{c}
z \\
w
\end{array}\right)=\left(\begin{array}{c}
z+J w \\
w
\end{array}\right) .
$$

We deduce that $X$ is invariant if and only if $W+J W \subseteq Z$. In particular, the only invariant subspace with $W$ equal to $\mathbb{F}_{p}^{n}$ is $\mathbb{F}_{p}^{2 n}$.

Clearly we may restrict our attention to invariant flags of proper subspaces. Based on Lemma 4.7, we define the fine type of an invariant flag $X_{0} \subset X_{1} \subset \cdots \subset X_{t}$ of proper subspaces to be $\left(d_{0}, \ldots, d_{t} ; \lambda^{0}, \ldots, \lambda^{t}\right)$, where $d_{i}=\operatorname{dim}\left(X_{i}\right)$, and $\lambda^{i}$ is the partition associated to $W_{i}$. Of course, the fine type of a flag determines its type. But, by Lemma 4.11, the number of flags of a given fine type is independent of $f$.

Lemma 4.11. The number of invariant flags $X_{0} \subset X_{1} \subset \cdots \subset X_{t}$ of proper subspaces with given fine type $\left(d_{0}, \ldots, d_{t} ; \lambda^{0}, \ldots, \lambda^{t}\right)$ does not depend on $f$.

Proof. An invariant subspace $X$ determines $W, Z$ and a linear map $\alpha: W \rightarrow \mathbb{F}_{p}^{n} / Z$ defined by $w+\alpha(w) \subseteq X \subseteq \mathbb{F}_{p}^{2 n}=\mathbb{F}_{p}^{n} \oplus \mathbb{F}_{p}^{n}$. Conversely, any such triple $W, Z, \alpha$ with $W+J W \subseteq Z$ determines an invariant $X$. For an invariant flag we also require that $W_{i} \subseteq W_{j}$ and $Z_{i} \subseteq Z_{j}$ for $i<j$; and that $\alpha_{i}(w)+Z_{j}=\alpha_{j}(w)$ for all $w \in W_{i}$.

By Corollary 4.10, the number of flags $W_{0} \subseteq W_{1} \subseteq \cdots \subseteq W_{t}$ with partition type $\left(\lambda^{0}, \ldots, \lambda^{t}\right)$ is independent of $f$. The number of flags $Z_{0} \subseteq \cdots \subseteq Z_{t}$ in $\mathbb{F}_{p}^{n}$ such that $W_{i}+J W_{i} \subseteq Z_{i}$ and $\operatorname{dim}\left(Z_{i}\right)=d_{i}-\operatorname{dim}\left(W_{i}\right)$ does not depend on the flag $W_{i}$ or on $f$ : for the type $\tau$ of the flag $W_{i}+J W_{i}$ is determined, and all flags of type $\tau$ are in the same orbit. Given flags $W_{i}$ and $Z_{i}$, the number of choices for the $\alpha_{i}$ is independent of $f$ : pick $\alpha_{1}$ first, and pick $\alpha_{i+1}$ to be any extension of $\alpha_{i}$.

Remark 4.12. Theorem 4.6 can be interpreted in terms of the prime ideals $\mathfrak{p}_{V}$ (see Remark 3.5). Let $V, W$ be elementary abelian subgroups of $G$ which are isomorphic in $\mathcal{A}_{\pi}$ but not conjugate in $G$. Then $\mathfrak{p}_{V} \cap S_{h}$ and $\mathfrak{p}_{W} \cap S_{h}$ are distinct prime ideals in $S_{h}$, but $\mathfrak{p}_{V} \cap S_{\pi}$ and $\mathfrak{p}_{W} \cap S_{\pi}$ are the same prime ideal of $S_{\pi}$. In the specific case constructed, $V, W$ have $p$-rank two and lie in an elementary abelian subgroup of rank $n^{2}$, the $p$-rank of $G$. Hence $\mathfrak{p}_{V}$ and $\mathfrak{p}_{W}$ have height $n^{2}-2$.

Remark 4.13. The authors believe that the categories $\mathcal{A}, \mathcal{A}_{h}$ and $\mathcal{A}_{\pi}$ are all distinct for the group $\mathrm{GL}_{m}\left(\mathbb{F}_{p}\right)$ for all sufficiently large $m$, whether odd or even. On the other hand, in the case when $m<4$, it can be shown that $\mathcal{A}=\mathcal{A}_{h}=\mathcal{A}_{\pi}$, except that $\mathcal{A} \neq \mathcal{A}_{h}$ when $m=3$ and $p$ is odd.

Acknowledgements. I.J.L. was partly funded by the Nuffield Foundation, grant no. SCI/180/96/127. 


\section{References}

1. J. L. Alperin, A Lie approach to finite groups, Groups-Canberra 1989 (ed. L. G. Kovács), pp. 1-9, Lecture Notes in Mathematics, no. 1456 (Springer, 1990).

2. L. Evens, The cohomology of groups (Clarendon, Oxford, 1991).

3. D. J. Green, On the cohomology of the sporadic simple group $J_{4}$, Math. Proc. Camb. Phil. Soc. 113 (1993), 253-266.

4. D. J. Green and I. J. Leary, The spectrum of the Chern subring, Commun. Math. Helv. 73 (1998), 406-426.

5. D. Quillen, The spectrum of an equivariant cohomology ring, II, Ann. Math. 94 (1971), $573-602$.

6. G. B. Segal and C. T. Stretch, Characteristic classes for permutation representations, Math. Proc. Camb. Phil. Soc. 90 (1981), 265-272.

7. B. B. Venkov, Cohomology algebras for some classifying spaces, Dokl. Akad. Nauk SSSR 127 (1959), 943-944. 